Maicon Sônego*

\title{
On the weakly degenerate Allen-Cahn equation
}

https://doi.org/10.1515/anona-2020-0004

Received September 15, 2018; accepted November 29, 2018.

Abstract: In this paper we consider a one-dimensional Allen-Cahn equation with degeneracy in the interior of the domain and Neumann boundary conditions. We allow the diffusivity coefficient vanish at some point of the space domain and we are addressed on the existence of stable non-constant solution.

Keywords: degenerate problem, Allen-Cahn equation, stable solution

MSc: Primary: 35A15; Secondary: 35B35, 35A01

\section{Introduction}

Consider the following semi-linear problem

$$
\left\{\begin{array}{l}
u_{t}(t, x)=\left(a(x) u_{x}(t, x)\right)_{x}+f(u(t, x)), \quad(t, x) \in \mathbb{R}^{+} \times(0,1) \\
u_{x}(t, 0)=u_{x}(t, 1)=0, \quad t \in \mathbb{R}^{+} \\
u(0, x)=u_{0}(x), \quad x \in(0,1)
\end{array}\right.
$$

where $f(u)=u-u^{3}$ and $a(\cdot) \in C([0,1])$ satisfies

(H1) $\quad a\left(x_{0}\right)=0$ at some $x_{0} \in(0,1)$;

(H2) $\quad a(x)>0$ for $x \in[0,1] \backslash\left\{x_{0}\right\}$ and

(H3) $1 / a \in L^{1}(0,1)$.

With these conditions, the parabolic problem (1.1) is called weakly degenerate (see [1], for instance) with degeneracy in the interior of the space domain. A typical example for $a(\cdot)$ satisfying the conditions (H1)-(H3) is given by $a(x)=\left|x-x_{0}\right|^{\alpha}(0<\alpha<1)$.

In this paper we are concerned to ensure the existence of solution to the evolution problem (1.1) and, mainly, to study the existence of stable solutions of the variational problem where the critical points of energy functional are stationary solutions of (1.1); that is

$$
\left\{\begin{array}{l}
\left(a(x) u_{x}(x)\right)_{x}+f(u)=0, x \in(0,1) \\
u_{x}(0)=u_{x}(1)=0
\end{array}\right.
$$

Roughly speaking, we study the role of the degeneracy of function $a(\cdot)$ at $x_{0}$ to the existence of a local minimizer of the energy functional associated to (1.2). This class of solutions (which we call stable solutions, see Definition 3.1 and Remark 3.2), in general, enjoys better qualitative and quantitative properties (monotonicity and symmetry, for instance) than the other solutions. In particular, when a minimizer is isolated - in this case we say asymptotically stable - it can describe the whole dynamics of the corresponding parabolic problem. We refer to the excellent monograph [2] for a comprehensive and complete presentation of the main results available on stable solutions.

*Corresponding Author: Maicon Sônego, Instituto de Matemática e Computação - Universidade Federal de Itajubá, MG, Brazil, E-mail: mcn.sonego@unifei.edu.br 
Degenerate problems have always attracted the attention of many authors, [1,3-7] and references therein. In these works it is assumed that the function $a(\cdot)$ degenerates at the boundary or in the interior of the space domain and the results are mainly related to the theory of control. In particular, in [1] the authors study degenerate parabolic problems with interior degeneracy, under Dirichlet boundary conditions, and show that under suitable assumptions, they generate analytic semi-groups. In addition, some applications to linear and semi-linear parabolic problems are provided. Similar results were achieved in [7], under Neumann boundary conditions, whose focus was to obtain Carleman estimates. With regard to existence of solution to the problem (1.1), the main results of the present work are based on these last two articles.

Here we will focus on a particular reaction term related to Allen-Cahn problem, $f(u)=u-u^{3}$. The AllenCahn equation has its origin in the theory of phase transitions ([8]) and it is used as a model for some nonlinear reaction-diffusion processes. For instance, assume that there are two populations $\mathcal{A}$ and $\mathcal{B}$ and that $u$ is a density measuring the percentage of the two populations at every point; that is, if $u(x)=1(u(x)=-1)$ at a point $x$, we have only population $\mathcal{A}$ (population $\mathcal{B}$ ) at $x$ and $u(x)=0$ means that at $x$ we have $50 \%$ of $\mathcal{A}$ and $50 \%$ of $\mathcal{B}$. The non-homogeneity of the medium is expressed by the space dependence of the diffusion coefficient $a(\cdot)$. If this coefficient vanishes at some point, then this will lead to the interruption of the migration and/or interaction of the species. Evidently, $u \equiv 1$ or $u \equiv-1$ are two stable states of the system, however, our goal is to obtain existence of stable non-constant solutions.

The study of existence or non-existence of stable solutions to semi-linear problems (in particular those of the Allen-Cahn type), with $a(x) \equiv 1$ or $a(\cdot)$ strictly positive, is the subject of numerous articles and books. In the face of an extensive literature, we cite [9-14] and references therein. Of course, allowing $a(\cdot)$ vanishes at some point brings with it several technical difficulties. Since the operator $A u:=\left(a u_{x}\right)_{x}$ is no longer elliptic (sometimes called degenerate elliptic), some basic analysis tools - such as the Maximum Principles, Hopf's Lemma and Spectral Theory - can not be used. To the best of our knowledge, the present work is the first to study the role of a degenerate diffusion coefficient related to the existence of stable solutions.

The technique we use here stems from the one presented in [11] (see also [13-15]). After finding a invariant set for the flux of (1.1) - hence the need to ensure the existence of solution - we use some variational techniques, in a convenient weighted Sobolev space, to show existence of a local minimizer of the energy functional in this invariant set.

Finally, let us remark that even our result of existence of solution to the problem (1.1), based on the works $[1,7]$, it is new and although it is essential to our main result, it may have interest of its own.

\section{Existence of solution}

In this section we deal with the existence of solution to the problem (1.1). It is important to note that the results of this section can easily be adapted to more general semi-linear equations.

Firstly, we should note that $A u=\left(a u_{x}\right)_{x}$, in a suitable domain, generates a analytic semigroup. For this purpose, we introduce the following weighted spaces (sometimes, we use ' to denote the derivative with respect to $x$ ):

$$
H_{a}^{1}(0,1):=\left\{u \in L^{2} ; u \text { absolutely continuous in }[0,1] \text { and } \sqrt{a} u^{\prime} \in L^{2}(0,1)\right\}
$$

with the norm

$$
\mid u\left\|_{H_{a}^{1}(0,1)}^{2}:=\right\| u\left\|_{L^{2}(0,1)}^{2}+\right\| \sqrt{a} u^{\prime} \|_{L^{2}(0,1)}^{2}
$$

and

$$
H_{a}^{2}(0,1):=\left\{u \in H_{a}^{1}(0,1) ; a u^{\prime} \in H^{1}(0,1)\right\}
$$

with

$$
\|u\|_{H_{a}^{2}(0,1)}^{2}:=\|u\|_{H_{a}^{1}(0,1)}^{2}+\left\|\left(a u^{\prime}\right)^{\prime}\right\|_{L^{2}(0,1)}^{2} .
$$

Definition 2.1. If $u_{0} \in L^{2}(0,1)$, a function $u$ is said to be a weak solution of (1.1) if

$$
u \in C\left([0, T] ; L^{2}(0,1)\right) \cap L^{2}\left(0, T ; H_{a}^{1}(0,1)\right)
$$


and

$$
\int_{0}^{1} u(T, x) \phi(T, x) d x-\int_{0}^{1} u_{0}(x) \phi(0, x) d x-\int_{(0, T) \times(0,1)} u \phi_{t} d x d t=-\int_{(0, T) \times(0,1)} a u_{x} \phi_{x} d x d t+\int_{(0, T) \times(0,1)} f(u) \phi d x d t
$$

for all $\phi \in H^{1}\left(0, T ; L^{2}(0,1)\right) \cap L^{2}\left(0, T ; H_{a}^{1}(0,1)\right)$.

Now we define the operator $A$ by $D(A):=\left\{u \in H_{a}^{2}(0,1) ; u^{\prime}(0)=u^{\prime}(1)=0\right\}$ and for any $u \in D(A), A u=\left(a u^{\prime}\right)^{\prime}$.

The proof of the next two results can be found in [1, Lemma 2.1] (see also [7, Lemma 2.1]) and [7, Theorem 2.1], respectively.

Lemma 2.2. For all $(u, v) \in D(A) \times H_{a}^{1}(0,1)$ one has

$$
\int_{0}^{1}\left(a u^{\prime}\right)^{\prime} v d x=-\int_{0}^{1} a u^{\prime} v^{\prime} d x
$$

Theorem 2.3. The operator $A: D(A) \rightarrow L^{2}(0,1)$ is self-adjoint, nonpositive on $L^{2}(0,1)$ and it generates an analytic contraction semigroup.

Now we proceed as in [1]. Since $A$ is a generator, and setting $B(t) u:=u$, working in the spaces considered above, we can prove that the problem below (with $c \in L^{\infty}\left(\mathbb{R}_{+} \times(0,1)\right)$ ) is well-posed in the sense of semigroup theory using some well-known perturbation technique (see [16], for instance).

$$
\left\{\begin{array}{l}
u_{t}(t, x)=\left(a(x) u_{x}(t, x)\right)_{x}+c(t, x) u(t, x), \quad(t, x) \in \mathbb{R}^{+} \times(0,1) \\
u_{x}(t, 0)=u_{x}(t, 1)=0, \quad t \in \mathbb{R}^{+} \\
u(0, x)=u_{0}(x), \quad x \in(0,1)
\end{array}\right.
$$

Hence, for a fixed $T>0$ we get the following result.

Theorem 2.4. If $c(\cdot, x) \in C^{1}\left(\mathbb{R}_{+}\right)$for all $x \in[0,1]$ and $u_{0} \in D(A)$ then there is a unique weak solution

$$
u \in H^{1}\left(0, T ; L^{2}(0,1)\right) \cap L^{2}\left(0, T ; H_{a}^{2}(0,1)\right) \cap C\left([0, T] ; H_{a}^{1}(0,1)\right)
$$

of (2.4) and

$$
\sup _{t \in[0, T]}\|u(t)\|_{H_{a}^{1}(0,1)}^{2}+\int_{0}^{T}\left(\left\|\frac{\partial u}{\partial t}\right\|_{L^{2}(0,1)}^{2}+\left\|\frac{\partial}{\partial x}\left(a \frac{\partial u}{\partial x}\right)\right\|_{L^{2}(0,1)}^{2}\right) d t \leq C\left\|u_{0}\right\|_{H_{a}^{1}(0,1)}^{2},
$$

for a positive constant $C$.

Next result can be found in [1, Theorem 5.4]

Lemma 2.5. The set $H^{1}\left(0, T ; L^{2}(0,1)\right) \cap L^{2}\left(0, T ; H_{a}^{2}(0,1)\right)$ is compactly imbedded in $C\left([0, T] ; L^{2}(0,1)\right) \cap$ $L^{2}\left(0, T ; H_{a}^{1}(0,1)\right)$.

We are now in position to state the main result of this section. The proof follows the steps of [1, Theorem 4.12], however, some modifications are necessary because we consider Neumann boundary conditions and the specific nonlinear term $f(u)=u-u^{3}$.

Theorem 2.6. If $u_{0}(x) \in H_{a}^{1}(0,1)$ then (1.1) has a solution

$$
u \in H^{1}\left(0, T ; L^{2}(0,1)\right) \cap L^{2}\left(0, T ; H_{a}^{2}(0,1)\right) .
$$


Proof. We set $X:=C\left([0, T] ; L^{2}(0,1)\right) \cap L^{2}\left(0, T ; H_{a}^{1}(0,1)\right)$ and for any $(x, v) \in(0,1) \times X, c^{v}(t, x):=$ $d(t, x, v(t, x))$ where $d(t, x, u)=1-u^{2}$. Now, we consider the function

$$
\mathcal{T}: v \in X \rightarrow u^{v} \in X,
$$

where $u^{v}$ is the unique solution of

$$
\left\{\begin{array}{l}
u_{t}(t, x)=\left(a(x) u_{x}(t, x)\right)_{x}+c^{v}(t, x) u(t, x), \quad(t, x) \in \mathbb{R}^{+} \times(0,1) \\
u_{x}(t, 0)=u_{x}(t, 1)=0, \quad t \in \mathbb{R}^{+} \\
u(0, x)=u_{0}(x), \quad x \in(0,1)
\end{array}\right.
$$

We use Theorem 2.4 to ensure that (2.7) has a unique weak solution $u \in X$. Now, we will prove that $\mathcal{T}$ has a fixed point $u^{v}$ (that is, $\mathcal{T}\left(u^{v}\right)=u^{v}$ ) to conclude that $u^{v}$ is a solution of (1.1).

By Schauder's Theorem, it is sufficient to prove that

(i) $\mathcal{T}: B_{X} \rightarrow B_{X}$,

(ii) $\quad \mathcal{T}$ is a compact function and

(iii) $\mathcal{T}$ is a continuous function,

where

$$
B_{X}:=\left\{v \in X ;\|v\|_{X} \leq C_{T}\left\|u_{0}\right\|_{L^{2}(0,1)}^{2}\right\},
$$

$C_{T}$ it is the same constant of Theorem 2.4 and

$$
\|v\|_{X}:=\sup _{t \in[0, T]}\left(\|u(t)\|_{L^{2}(0,1)}^{2}\right)+\int_{0}^{T}\left\|\sqrt{a} u_{X}\right\|_{L^{2}(0,1)}^{2} d t .
$$

The items (i) and (ii) are consequence of Theorem 2.4 and Lemma 2.5, respectively.

To prove (iii) we take $v_{k} \in X$ such that $v_{k} \rightarrow v$ in $X$, as $k \rightarrow \infty$. We will prove that $\mathcal{T}\left(v_{k}\right)=u^{v_{k}}:=$ $u^{k} \rightarrow \mathcal{T}(v)=u^{v}$ in $X$, as $k \rightarrow \infty$. Recall that $u^{k}$ and $u^{v}$ are the solutions of (2.7) associated to $v_{k}$ and $v$, respectively. As $D(A)$ is dense in $H_{a}^{1}(0,1)$ ([7]), (2.5) and (2.6) occurs for $u_{0} \in H_{a}^{1}(0,1)$. Hence, $u^{k} \in B_{Y}$ where $Y=H^{1}\left(0, T ; L^{2}(0,1)\right) \cap L^{2}\left(0, T ; H_{a}^{2}(0,1)\right)$ and, up to a sub-sequence, $u^{k}$ converges weakly to some $\bar{u}$ in $Y$. By Lemma $2.5, u^{k}$ converges strongly to $\bar{u}$ in $X$.

Multiplying the equation

$$
u_{t}^{k}(t, x)=\left(a(x) u_{x}^{k}(t, x)\right)_{x}+c^{v^{k}}(t, x) u^{k}(t, x)
$$

by a test function $\phi \in H^{1}\left(0, T ; L^{2}(0,1)\right) \cap L^{2}\left(0, T ; H_{a}^{1}(0,1)\right)$ and integrating over $(0, T) \times(0,1)$ (recall the Lemma 2.2) we get

$$
\begin{gathered}
\int_{0}^{1} u^{k}(T, x) \phi(T, x) d x-\int_{0}^{1} u_{0}(x) \phi(0, x) d x-\int_{0}^{T} \int_{0}^{1} \phi_{t}(t, x) u^{k}(t, x) d x d t \\
=\int_{0}^{T} a(x) u_{x}^{k}(t, 1) \phi(t, 1) d t-\int_{0}^{T} a(x) u_{x}^{k}(t, 0) \phi(t, 0) d t \\
-\int_{0}^{T} \int_{0}^{1} a(x) u_{x}^{k}(t, x) \phi_{x}(t, x) d x d t+\int_{0}^{T} \int_{0}^{1} c^{v^{k}}(t, x) u^{k}(t, x) \phi(t, x) d x d t .
\end{gathered}
$$

We recall that $u_{x}^{k}(t, 1)=u_{x}^{k}(t, 0)=0$ and our next step is to prove that

(a) $\lim _{k \rightarrow \infty} \int_{0}^{1} u^{k}(T, x) \phi(T, x) d x=\int_{0}^{1} \bar{u}(T, x) \phi(T, x) d x$; 
(b) $\quad \lim _{k \rightarrow \infty} \int_{0}^{T} \int_{0}^{1} \phi_{t}(t, x) u^{k}(t, x) d x d t=\int_{0}^{T} \int_{0}^{1} \phi_{t}(t, x) \bar{u}(t, x) d x d t$;

(c) $\quad \lim _{k \rightarrow \infty} \int_{0}^{T} \int_{0}^{1} a(x) u_{x}^{k}(t, x) \phi_{x}(t, x) d x d t=\int_{0}^{T} \int_{0}^{1} a(x) \bar{u}_{x}(t, x) \phi_{x}(t, x) d x d t$;

(d) $\quad \lim _{k \rightarrow \infty} \int_{0}^{T} \int_{0}^{1} c^{v^{k}}(t, x) u^{k}(t, x) \phi(t, x) d x d t=\int_{0}^{T} \int_{0}^{1} c^{v}(t, x) \bar{u}(t, x) \phi(t, x) d x d t$.

Since $u^{k}$ converges strongly to $\bar{u}$ in $X$, it is immediate to prove (a)-(c). In order to prove (d) we recall that $c^{v^{k}}(t, x)=1-\left(v^{k}\right)^{2}(t, x)$ and $v^{k}$ converges strongly to $v$ in $X$. Therefore, we can conclude that $v^{k}$ converges to $v$ a.e. as well as $u^{k}$ converges to $\bar{u}$ a.e.. Thus,

$$
\begin{aligned}
& \left|\int_{0}^{T} \int_{0}^{1} c^{v^{k}}(t, x) u^{k}(t, x) \phi(t, x)-c^{v}(t, x) \bar{u}(t, x) \phi(t, x) d x d t\right| \\
& \leq \int_{0}^{T} \int_{0}^{1}\left|\left(u^{k}(t, x)-\bar{u}(t, x)\right) c^{v^{k}}(t, x) \phi(t, x)\right| d x d t \\
& +\int_{0}^{T} \int_{0}^{1}\left|\left(c^{v^{k}}(t, x)-c^{v}(t, x)\right) \bar{u}(t, x) \phi(t, x)\right| d x d t \\
& =\int_{0}^{T} \int_{0}^{1}\left|u^{k}(t, x)-\bar{u}(t, x)\right|\left|\left(1-\left(v^{k}\right)^{2}(t, x)\right) \phi(t, x)\right| d x d t \\
& +\int_{0}^{T} \int_{0}^{1}\left|\left(v^{k}\right)^{2}(t, x)-v^{2}(t, x)\right||\bar{u}(t, x) \phi(t, x)| d x d t,
\end{aligned}
$$

and (d) holds by an application of Lebesgue Theorem.

We proved that $\bar{u}$ is the unique weak solution of (2.4) in $Y \subset X$ associated to $v$; that is $\bar{u}=u^{v}$ and (iii) is proved. It follows that $\mathcal{T}$ has a fixed point $u^{v} \in Y$ which is a solution of (1.1). The theorem is proved.

\section{Existence of stable solutions}

We start by defining an energy functional $E: H_{a}^{1}(0,1) \rightarrow \mathbb{R}$ by

$$
E(u)=\frac{1}{2} \int_{0}^{1} a(x)\left(u^{\prime}(x)\right)^{2} d x-\int_{0}^{1} F(u(x)) d x
$$

where

$$
F(u):=\int_{0}^{u}\left[s-s^{3}\right] d s=\frac{u^{2}}{2}-\frac{u^{4}}{4} .
$$

It is not difficult to verify that $E$ is twice continuously differentiable and a simple computation give us that its critical points are weak stationary solutions of (1.1) (i.e. weak solutions of (1.2)). 
Definition 3.1. Let $u$ be a weak solution to (1.2). We say that $u$ is stable if

$$
\int_{0}^{1} a\left(\phi^{\prime}\right)^{2} d x-\int_{0}^{1} f^{\prime}(u) \phi^{2} d x \geq 0
$$

for any $\phi \in H_{a}^{1}(0,1)$.

Remark 3.2. Note that (3.2) is equivalent to the second variation of the energy functional $E(\cdot)$ at $u$ to be nonnegative. Therefore, local minimizers of $E$ are stable solutions of (1.2).

In order to state our next results, we set $I_{l}, I_{r}$ two sub-intervals of $(0,1)$ such that $\overline{I_{l}} \subset\left(0, x_{0}\right)$ and $\overline{I_{r}} \subset\left(x_{0}, 1\right)$.

Lemma 3.3 (Poincaré-type inequality). There exists a constant $\mathcal{C}_{j}(j=l, r)$, depending only on $I_{j}$ and $\left.a\right|_{I_{j}}$, such that

$$
\int_{I_{j}}\left(u-\overline{u^{j}}\right)^{2} d x \leq \mathcal{C}_{j} \int_{I_{j}} a\left(u^{\prime}\right)^{2} d x
$$

for each function $u \in H_{a}^{1}\left(I_{j}\right)$, where $\overline{u^{j}}=\frac{1}{\left|I_{j}\right|} \int_{I_{j}} u d x$.

Proof. For brevity, we omit the sub-indices $j=l, r$. We argue by contradiction; that is, we suppose that for each $k \in \mathbb{N}$, there exists $u_{k} \in H_{a}^{1}(0,1)$ such that

$$
\left\|u_{k}-\overline{u_{k}}\right\|_{L^{2}(I)}>k\left\|\sqrt{a} u_{k}^{\prime}\right\|_{L^{2}(I)},
$$

where $\overline{u_{k}}:=\frac{1}{|I|} \int_{I} u_{k} d x$.

We renormalize by defining

$$
v_{k}:=\frac{u_{k}-\overline{u_{k}}}{\left\|u_{k}-\overline{u_{k}}\right\|_{L^{2}(I)}}, \quad k \in \mathbb{N} .
$$

It follows that $\overline{v_{k}}:=\frac{1}{|I|} \int_{I} v_{k} d x=0,\left\|v_{k}\right\|_{L^{2}(I)}=1$ and by (3.4)

$$
\left\|\sqrt{a} v_{k}^{\prime}\right\|_{L^{2}(I)}<\frac{1}{k}, \quad k \in \mathbb{N} .
$$

In particular the functions $\left\{v_{k}\right\}_{k \in \mathbb{N}}$ are bounded in $H_{a}^{1}(I)$. As $H_{a}^{1}(I)$ is compactly imbedded in $L^{2}(I)$ (the proof is analogous to that present in [1, Theorem 5.1]), there exist a sub-sequence $\left\{v_{k_{j}}\right\}_{j \in \mathbb{N}}$ and a function $v \in L^{2}(I)$ such that

$$
v_{k_{j}} \rightarrow v \text { in } L^{2}(I)
$$

From (3.5)

$$
\bar{v}:=\frac{1}{|I|} \int_{I} v d x=0 \text { and }\|v\|_{L^{2}(I)}=1 .
$$

If $\phi \in C_{0}^{\infty}(I)$ we use (3.6) to conclude that

$$
\int_{I} v \phi^{\prime} d x=\lim _{j \rightarrow \infty} \int_{I} v_{k_{j}} \phi^{\prime} d x=-\lim _{j \rightarrow \infty} \int_{I} \sqrt{a} v_{k_{j}}^{\prime} \frac{1}{\sqrt{a}} \phi d x=0 .
$$

Hence $v \in H^{1}(I)$ and $v^{\prime}=0$ a.e. in $I$. It follows that $v$ is constant in $I$ which is a contradiction with (3.8).

Now, we set the positive number

$$
\epsilon_{0}:=\frac{1}{4} \min \left\{\left|I_{l}\right| \min \left\{1,\left(1 / \mathcal{C}_{l}\right)\right\},\left|I_{r}\right| \min \left\{1,\left(1 / \mathcal{C}_{r}\right)\right\}\right\}
$$

where $C_{j}(j=l, r)$ is the optimal constant in (3.3).

Our main result is stated as follow. 
Theorem 3.4. If there is $\delta>0$ such that $\delta<2 \epsilon_{0}, Q_{\delta}:=\left[x_{0}-\delta, x_{0}+\delta\right] \cap\left(\overline{I_{l}} \cup \overline{I_{r}}\right)=\emptyset$ and

$$
\max _{x \in Q_{\delta}}\{a(x)\}<\frac{\delta}{2}\left(\epsilon_{0}-\frac{\delta}{2}\right)
$$

then (1.2) admits a non-constant stable solution.

For each $t>0$, we consider

$$
T(t): H_{a}^{1}(0,1) \rightarrow H_{a}^{1}(0,1)
$$

defined by

$$
T(t)\left[u_{0}(x)\right]=u(t, x)
$$

where $u(t, x)$ is the solution of (1.1) with $u(0, x)=u_{0}(x)$ given by Theorem 2.6. Then $t \rightarrow T(t)\left[u_{0}(x)\right]$ is continuous for any $u_{0} \in H_{a}^{1}(0,1)$. Moreover, $T(t)$ is a compact operator because $T(t)\left[u_{0}(x)\right] \in H_{a}^{2}(0,1)$ and $H_{a}^{2}(0,1)$ is compactly imbedded in $H_{a}^{1}(0,1)$ (see [1, Theorem 5.2]). For simplicity, we denote $T(t)\left[u_{0}(x)\right]$ by $T(t) u_{0}$.

Proposition 3.5. Consider the set

$$
\Lambda\left(I_{l}, I_{r}\right)=\left\{\begin{array}{c}
v \in H_{a}^{1}(0,1) ;-1 \leq v(x) \leq 1 \forall x \in[0,1], \\
\int_{I_{l}} v<0, \int_{I_{r}} v>0, E(v)<\epsilon_{0}-\frac{1}{4}
\end{array}\right\} .
$$

If $u_{0} \in \Lambda$ then $T(t) u_{0} \in \Lambda$ for all $t>0$.

Proof. Claim 1: $-1 \leq T(t) u_{0}=u(t, x) \leq 1$ in $[0,1]$ for all $t>0$.

If there is $(\bar{t}, \bar{x})$ such that $u(\bar{t}, \bar{x})>1$ then if $T>\bar{t}$

$$
u_{M}:=\max _{(0, T] \times[0,1]} u(t, x)>1
$$

and $u_{M}=u(\tilde{t}, \tilde{x})$ at some $(\tilde{t}, \tilde{x}) \in(0, T] \times[0,1]$. We have three possibilities:

(i) $\tilde{x} \in(0,1) \backslash\left\{x_{0}\right\}$,

(ii) $\tilde{x}=x_{0}$ or

(iii) $\tilde{x} \in\{0,1\}$.

If (i) holds we can apply the Maximum Principle in a sub-interval that does not contain $x_{0}$. Hence, we have a contradiction since $u(0, x)=u_{0}(x) \leq 1$. For (iii), we use a one-dimensional version of Hopf's Lemma and that $u_{x}(\tilde{t}, 0)=u_{x}(\tilde{t}, 1)=0$ to get a contradiction. Finally, we note that

$$
u_{t}\left(t, x_{0}\right)=u\left(t, x_{0}\right)-u^{3}\left(t, x_{0}\right), t>0
$$

and then $u_{t}\left(t, x_{0}\right)<0$ for $t$ near of $\tilde{t}$. It follows that (ii) does not occur. Therefore $u(t, x) \leq 1(t>0)$ and analogously we prove $-1 \leq u(t, x)$ for all $t>0$ which proves the Claim 1 .

Claim 2: $E(u(t, x))<\epsilon_{0}-\frac{1}{4}$.

Indeed, it is true because

$$
\frac{d}{d t} E(u(t, x))=-\int_{0}^{1}\left(u_{t}(t, x)\right)^{2} d x<0 \text { and } E(u(0, x))<\epsilon_{0}-\frac{1}{4} .
$$

Claim 3: $\int_{I_{l}} u(t, x) d x<0$ and $\int_{I_{r}} u(t, x) d x>0$ for all $t>0$.

By contradiction, let $t_{1}>0$ be such that $u_{1}(x):=u\left(t_{1}, x\right)$ satisfies

$$
\int_{I_{l}} u_{1}(x) d x=0
$$


Then, by Lemma 3.3

$$
\int_{I_{l}} u_{1}^{2} d x \leq \mathcal{C}_{l} \int_{I_{l}} a\left(u_{1}^{\prime}\right)^{2} d x
$$

Now, note that

$$
\begin{gathered}
0 \geq f(s) \geq s, \text { for }-1 \leq s \leq 0 \\
0 \leq f(s) \leq s \text { for } 0 \leq s \leq 1
\end{gathered}
$$

Hence,

$$
F\left(u_{1}\right)=\int_{0}^{u_{1}} f(s) d s \leq \int_{0}^{u_{1}} s d s=\frac{\left(u_{1}\right)^{2}}{2}
$$

and

$$
\begin{aligned}
E\left(u_{1}\right) & =\frac{1}{2} \int_{0}^{1} a\left(u_{1}^{\prime}\right)^{2} d x-\int_{0}^{1} F\left(u_{1}\right) d x \\
& \geq \frac{1}{2 \mathcal{C}_{l}} \int_{I_{l}}\left(u_{1}\right)^{2} d x-\int_{0}^{1} F\left(u_{1}\right) d x \\
& \geq \frac{1}{\mathcal{C}_{l}} \int_{I_{l}} F\left(u_{1}\right) d x-\int_{I_{l}} F\left(u_{1}\right) d x-\int_{(0,1) \backslash I_{l}} F\left(u_{1}\right) d x .
\end{aligned}
$$

We also have

$$
E\left(u_{1}\right) \leq E\left(u_{0}\right)<\epsilon_{0}-\frac{1}{4} .
$$

Therefore, as $F\left(u_{1}\right) \leq F(1)=1 / 4$

$$
\epsilon_{0}>\left[\frac{1}{\varrho_{l}}-1\right] \int_{I_{l}} F\left(u_{1}\right) d x-\int_{(0,1) \backslash I_{l}} F\left(u_{1}\right) d x+\frac{1}{4}>\left[\frac{1}{\varrho_{l}}-1\right] \int_{I_{l}} F\left(u_{1}\right) d x+\left|I_{l}\right| \frac{1}{4}
$$

and

- if $\frac{1}{\mathfrak{C}_{l}} \geq 1$ then $\epsilon_{0}>\frac{\left|I_{l}\right|}{4}$ or

- if $\frac{1}{\mathfrak{C}_{l}}<1$ then $\epsilon_{0}>\frac{\left|I_{l}\right|}{4 \mathcal{C}_{l}}$.

In both cases we have a contradiction. Similarly we prove that $\int_{I_{r}} u(t, x) d x>0$ for all $t>0$. It is proved that $\Lambda$ is invariant under $T(t)$ for $t \geq 0$.

Proposition 3.6. If $\Lambda \neq \emptyset$ then (1.1) has at least one non-constant stationary solution $u \in \Lambda$ which is stable in $H_{a}^{1}(0,1)$.

Proof. If $v \in \Lambda$, by Proposition 3.5, $y(v):=\{T(t) v ; t \geq 0\} \subset \Lambda$ and because the system is gradient ( $E$ is a functional of Lyapunov) $y(v)$ is compact. It follows that

$$
\omega(v):=\left\{\lim _{t_{n} \rightarrow \infty} T\left(t_{n}\right) v=u, \text { for some real sequence }\left(t_{n}\right)\right\} \neq \emptyset .
$$

If $\mathcal{E}$ is the set of all equilibrium solutions to (1.1) then $\omega(v) \subset \mathcal{E}$. Hence, if $u \in \omega(v)$ then $-1 \leq u \leq 1$, $E(u) \leq E(v)<\epsilon_{0}-(1 / 4)$ and, as before, it is possible to prove that $\int_{I_{l}} u<0, \int_{I_{r}} u>0$; that is, $\omega(v) \subset \Lambda$. 
Therefore, if $v \in \Lambda, \bar{\Lambda} \cap \mathcal{\varepsilon} \neq \emptyset$. Moreover $\bar{\Lambda} \cap \mathcal{E}$ is compact in $H_{a}^{1}(0,1)$. Indeed, we note that $\bar{\Lambda} \cap \mathcal{\varepsilon}$ is bounded in $H_{a}^{1}(0,1)$ because for any $u \in \bar{\Lambda} \cap \mathcal{E},-1 \leq u \leq 1$ and

$$
a(x) u^{\prime}(x)=\int_{0}^{x} u^{3}(s)-u(s) d s, \forall x \in(0,1) \text { (see (2.2)). }
$$

It is not difficult to see that $\mathcal{E}$ is closed in $H_{a}^{1}(0,1)$. Now, as $T(t)[\bar{\Lambda} \cap \mathcal{E}]=\bar{\Lambda} \cap \mathcal{E}$ and $T(t)$ is a compact operator, we conclude that $\bar{\Lambda} \cap \mathcal{E}$ is compact in $H_{a}^{1}(0,1)$.

Because of the continuity of $E$, there is $e_{0} \in \bar{\Lambda} \cap \mathcal{E}$ such that $E\left(e_{0}\right) \leq E(v)$ for all $v \in \bar{\Lambda} \cap \mathcal{E}$. More than that, $E\left(e_{0}\right) \leq E(v)$ for all $v \in \Lambda$ since otherwise there would be $v_{1} \in \Lambda$ such that $E\left(v_{1}\right)<E\left(e_{0}\right)$. As before $\omega\left(v_{1}\right) \subset \Lambda$ and then, for all $v \in \omega\left(v_{1}\right)$,

$$
E(v) \leq E\left(v_{1}\right)<E\left(e_{0}\right)
$$

which is a contradiction.

The next step is to prove that $e_{0}$ is a local minimum of $E$ in $H_{a}^{1}(0,1)$. Let $\Lambda_{j}(j=1, \ldots, 4)$ be the sets

- $\quad \Lambda_{1}:=\left\{u \in H_{a}^{1}(0,1) ;-1<u<1\right.$ a.e. in $\left.(0,1)\right\}$;

- $\Lambda_{2}:=\left\{u \in H_{a}^{1}(0,1) ; \int_{I_{l}} u<0\right\}$;

- $\Lambda_{3}:=\left\{u \in H_{a}^{1}(0,1) ; \int_{I_{r}} u>0\right\}$;

- $\Lambda_{4}:=\left\{u \in H_{a}^{1}(0,1) ; E(u)<\epsilon_{0}-\frac{1}{4}\right\}$.

Claim 1: $\cap_{j=1}^{4} \Lambda_{j}$ is an open set in $H_{a}^{1}(0,1)$.

Indeed, $\Lambda_{j}(j=2, \ldots, 4)$ are open in $H_{a}^{1}(0,1)$ by the continuity of the functionals $E, E_{l}(u):=\int_{I_{l}} u$ and $E_{r}(u):=\int_{I_{r}} u$ in $H_{a}^{1}(0,1)$. It is not difficult to prove that $\Lambda_{1}$ is open in $H_{a}^{1}(0,1)$ using that $H_{a}^{1}(0,1) \hookrightarrow C(0,1)$. Claim 1 is proved.

Claim 2: $e_{0} \in \cap_{j=1}^{4} \Lambda_{j}$. By an application of Maximum Principle (recall that $e_{0} \in \mathcal{E}$ ), it is possible to conclude that $-1<e_{0}(x)<1$ for all $x \in(0,1) \backslash\left\{x_{0}\right\}$. This implies that $e_{0} \in \Lambda_{1}$. Clearly, $\int_{I_{l}} e_{0} \leq 0$ and $\int_{I_{r}} e_{0} \geq 0$ and if the equality occurs we get a contradiction as before. Thus $e_{0} \in \Lambda_{1} \cap \Lambda_{2}$. We have that $E\left(e_{0}\right) \leq \epsilon_{0}-(1 / 4)$ and if $E\left(e_{0}\right)=\epsilon_{0}-(1 / 4)$ then for any $v \in \Lambda$,

$$
E(v)<\epsilon_{0}-(1 / 4)=E\left(e_{0}\right)
$$

which contradicts $E\left(e_{0}\right) \leq E(v)$ for all $v \in \Lambda$. Therefore $e_{0} \in \Lambda_{4}$ and this proves Claim 2. Thus, $e_{0}$ is a local minimum of $E$ in $H_{a}^{1}(0,1)$ and then

$$
E^{\prime \prime}\left(e_{0}\right) \phi \geq 0 \forall \phi \in H_{a}^{1}(0,1)
$$

i.e.

$$
\int_{0}^{1}\left[a(x)\left(\phi^{\prime \prime}(x)\right)^{2}-f^{\prime}\left(e_{0}\right) \phi^{2}\right] d x \geq 0 \quad \forall \phi \in H_{a}^{1}(0,1)
$$

which proves that $e_{0}$ is a stable non-constant $\left(e_{0} \in \Lambda\right)$ solution of (1.2).

Finally, we are in position to prove our main result.

Proof of the Theorem 3.4. We shall prove that $\Lambda \neq \emptyset$ and the theorem follows by Proposition 3.6.

Consider the signed distance function defined in $\mathbb{R}$ by

$$
d\left(x, x_{0}\right)= \begin{cases}\left|x-x_{0}\right|, & x \geq x_{0} \\ -\left|x-x_{0}\right|, & x<x_{0}\end{cases}
$$

and $\xi: \mathbb{R} \rightarrow \mathbb{R}$ defined by

$$
\xi(t)= \begin{cases}-1, & t \leq-\delta \\ \frac{t}{\delta}, & -\delta<t<\delta \\ 1, & t \geq \delta\end{cases}
$$


We will show that $w_{0}(x):=\left.\xi\left(d\left(x, x_{0}\right)\right)\right|_{(0,1)} \in \Lambda$. It is not difficult to see that $w_{0} \in H_{a}^{1}(0,1) ;-1 \leq w_{0} \leq 1$; $\int_{I_{l}} w_{0}<0$ and $\int_{I_{r}} w_{0}>0$.

Now, if we set $a_{M}^{\delta}:=\max _{x \in Q_{\delta}}\{a(x)\}$ and recalling that $\left(d^{\prime}\left(x, x_{0}\right)\right)^{2}=1$ and $F(1)=F(-1)=1 / 4$,

$$
\begin{aligned}
E\left(w_{0}\right) & =\frac{1}{2} \int_{0}^{1} a(x)\left(w_{0}^{\prime}(x)\right)^{2} d x-\int_{0}^{1} F\left(w_{0}(x)\right) d x \\
& =\frac{1}{2} \int_{Q_{\delta}} a(x)\left(w_{0}^{\prime}(x)\right)^{2} d x-\int_{Q_{\delta}} F\left(w_{0}(x)\right) d x-\frac{(1-2 \delta)}{4} \\
& \leq a_{M}^{\delta} \frac{1}{\delta^{2}}\left|Q_{\delta}\right|-\frac{(1-2 \delta)}{4} \\
& =a_{M}^{\delta} \frac{2}{\delta}+\frac{\delta}{2}-\frac{1}{4} .
\end{aligned}
$$

By (3.10), $E\left(w_{0}\right)<\epsilon_{0}-(1 / 4)$ and then $w_{0} \in \Lambda$ which proves the Theorem 3.4.

Remark 3.7. It is common to say that the solution $e_{0}$ obtained in this work "is trapped at the bottom of an energy well". That is why we say that it is stable. A natural question is whether such a solution is asymptotically stable; that is, to know if the solutions of the corresponding parabolic problem (problem (1.1)) with the initial data near $e_{0}$ tend to $e_{0}$ as $t \rightarrow \infty$. This type of stability - also called linearized stability or Lyapunov stability - in general, it is accomplished by studying the spectrum of the corresponding linearized problem. However, the degeneracy considered here makes it impossible to carry out such a study. This question has been studied when the degenerate operator has an uniformly elliptic direction; which, obviously, the operator considered here does not have. For all the details on this issue, we cite [17].

Remark 3.8. In the case where $A=\left(a u_{x}\right)_{x}$ is strongly degenerate; that is, $a \in W^{1, \infty}(0,1)$ satisfies (H1) and (H2) but $1 / a \notin L^{1}(0,1)$, can not be carried out in an analogous way. The hypotheses (H1)-(H3) are essential in all results of this work which makes the strongly degenerate case an open problem.

\section{References}

[1] G. Fragnelli, G. R. Goldstein, J. A. Goldstein, and S. Romanelli, Generators with interior degeneracy on spaces of $L^{2}$ type, Electron. J. Differential Equations. (2012) 1-30.

[2] L. Dupaigne, Stable Solutions of Elliptic Partial Differential Equations, CRC press, 2011.

[3] P. Cannarsa, G. Fragnelli, and J. Vancostenoble, Regional controllability of semilinear degenerate parabolic equations in bounded domains,Journal of Mathematical Analysis and Applications. (2), (2006), 320, 804-818.

[4] P. Caldiroli, R. Musina, Existence and nonexistence results for a class of nonlinear, singular sturm-liouville equations, Advances in Differential Equations. (3), (2001), 6, 303-326.

[5] P. Caldiroli, R. Musina, On a variational degenerate elliptic problem, Nonlinear Differential Equations and Applications NoDEA. (2), (2000), 7, 187-199.

[6] F. Alabau-Boussouira, P. Cannarsa and G. Fragnelli, Carleman estimates for degenerate parabolic operators with applications to null controllability, Journal of Evolution Equations. (2), (2006), 6, 161-204.

[7] I. Boutaayamou, G. Fragnelli and L. Maniar, Carleman estimates for parabolic equations with interior degeneracy and Neumann boundary conditions, Journal d'Analyse Mathématique. (1), (2018), 135, 1-35.

[8] S. M. Allen, J. W. Cahn, A microscopic theory for antiphase boundary motion and its application to antiphase domain coarsening, Acta Metallurgica. (6), (1979), 27, 1085-1095.

[9] G. Fusco, J. K. Hale, Stable equilibria in a scalar parabolic equation with variable diffusion, SIAM Journal on Mathematical Analysis. (6), (1985), 16, 1152-1164.

[10] K. Kurata, H. Matsuzawa. Multiple stable patterns in a balanced bistable equation with heterogeneous environments, Applicable Analysis. (7), (2010), 89, 1023-1035. 
[11] H. Matano, Asymptotic behavior and stability of solutions of semilinear diffusion equations, Publications of the Research Institute for Mathematical Sciences. (2), 15, (1979), 401-454.

[12] A. S. do Nascimento, Bifurcation and stability of radially symmetric equilibria of a parabolic equation with variable diffusion, Journal of Differential Equations. (1), (1989), 77, 84-103

[13] A. S. do Nascimento, On the role of diffusivity in some stable equilibria of a diffusion equation, Journal of Differential Equations. (2), (1999), 155, 231-244.

[14] J. C. Simal, A. S. do Nascimento and Maicon Sônego, Sufficient conditions on diffusivity for the existence and nonexistence of stable equilibria with nonlinear flux on the boundary, Electronic Journal of Differential Equations. (2012), 1-14.

[15] N. Consul, J. Solá-Morales, Stable nonconstant equilibria in parabolic equations with nonlinear boundary conditions, Comptes Rendus de l’Académie des Sciences. Série 1, Mathématique.(3), (1995), 321, 299-304.

[16] J. A. Goldstein, Semigroups of Linear Operators and Applications. Oxford Univ. Press, Oxford, New York, 1985.

[17] D. D. Monticelli, K. R. Payne, Maximum principles for weak solutions of degenerate elliptic equations with a uniformly elliptic direction, Journal of Differential Equations. (7), (2009), 247, 1993-2026. 